\title{
Time-Domain Variability Analysis of General Linear Systems Terminated with Nonlinear Devices
}

\author{
Yinghao Ye, Domenico Spina, Paolo Manfredi, Dries Vande Ginste, Tom Dhaene \\ IDLab, Department of Information Technology, Ghent University - imec, Gent, Belgium. \\ Email: yinghao.ye@ugent.be
}

\begin{abstract}
In this paper, a new approach for the time-domain variability analysis of general linear systems terminated with nonlinear devices is presented. A deterministic, stable and passive model of the stochastic system under study is built starting from the Polynomial Chaos (PC) coefficients of the system's scattering parameters, and by means of the Galerkin projection (GP) method and the Vector Fitting (VF) algorithm. This model is then converted into an equivalent circuit via a suitable synthesis technique. The nonlinear terminations with stochastic input and output signals are also represented by SPICE-compatible equivalent models, based on the stochastic testing (ST) method. Finally, the equivalent models for both the linear and nonlinear parts are suitably connected and analyzed with a standard circuit simulator. Only a single time-domain simulation is necessary to compute the PC model of the port voltages and currents. The proposed approach, which is applicable to any passive system describable with a scattering representation, is here validated against state-of-the-art techniques based on the simulation of a distributed transmission-line network with diodes and nonlinear drivers.
\end{abstract}

Index Terms-Polynomial Chaos, nonlinear, variability analysis, passivity.

\section{INTRODUCTION}

In the recent years, the increasing integration and miniaturization of integrated circuits (ICs) have prompted the scientific community to dedicate a great deal of attention in studying the effects of geometrical and/or electrical parameter variations on the performance of modern designs, which are largely affected by the tolerances of the manufacturing process. The Monte Carlo (MC) analysis represents the standard approach for variability analysis, thanks to its robustness and accuracy, but since it requires to perform a large number of simulations, it is also time and resource intensive.

An efficient and accurate alternative is represented by techniques based on the PC expansion [1]-[7], which models a stochastic process as a summation of orthogonal basis functions in the random variable under study, with suitable coefficients. The interested reader may consult [1]-[9] for a reference to PC theory and applications. In particular, a technique able to compute a stable and passive model for the time-domain variability analysis of generic linear systems has been recently proposed in the literature [7]. It is based on the computation of the PC coefficients of the scattering parameters of the system under study, on the GP method [2], [9], and on the VF algorithm [10], [11], and it is readily applicable to a wide range of different microwave systems (i.e. connectors, distributed filters, interconnections).
However, the technique in [7] can only tackle the case of linear port terminations. In the proposed contribution, we overcome this limitation by incorporating the equivalent models of generic complex nonlinear devices as described in [6]. The combination of the techniques in [6] and [7] results in a novel and powerful method for the variability analysis of complex high-speed links or microwave circuits, which can consist of different elements (lumped, distributed, passive and active ones). Furthermore, the proposed method is applicable to systems requiring full-wave simulations and, once the models for the linear and nonlinear parts are built, the variability analysis of the entire circuit can be performed directly in the time domain by means of standard SPICE-type circuit simulators, avoiding the circuit-EM co-simulations.

This paper is structured as follow: Section II describes the novel proposed approach. A suitable numerical example is presented in Section III in order to illustrate the performance of the proposed method. Conclusions are drawn in Section IV.

\section{Stochastic Macromodeling of Complex MicROWAVE SYSTEMS}

For simplicity, in the rest of this paper we will consider the case where the random variables are independent and the focus will be on deterministic nonlinear elements, whose input signals are however under the effect of stochastic variations arising from the linear part. It should be noted that it is possible to extend the proposed method to the case of correlated random variables [8], [9]. Furthermore, orthonormal PC basis functions are considered.

The key idea of the proposed approach is to compute equivalent circuits that describe the behavior of the PC coefficients of the voltages and currents at the ports of the linear and nonlinear components, starting from the original stochastic circuit. Section II-A describes the method adopted for the linear elements of the circuit, while Section II-B discusses the modeling of the nonlinear components.

\section{A. Stochastic Macromodeling of General Passive Linear Sys-} tems

When a linear system is subjected to stochastic effects caused by the random variation of geometric or electrical parameters, here denoted by $\boldsymbol{\xi}$, the relationship between the $\boldsymbol{\xi}$ dependent incident $\boldsymbol{a}(\boldsymbol{\xi}) \in \mathbb{C}^{N \times 1}$ and reflected $\boldsymbol{b}(\boldsymbol{\xi}) \in \mathbb{C}^{N \times 1}$ waves at the $N$ system ports becomes

$$
\boldsymbol{b}(\boldsymbol{\xi})=\boldsymbol{S}(\boldsymbol{\xi}) \boldsymbol{a}(\boldsymbol{\xi})
$$




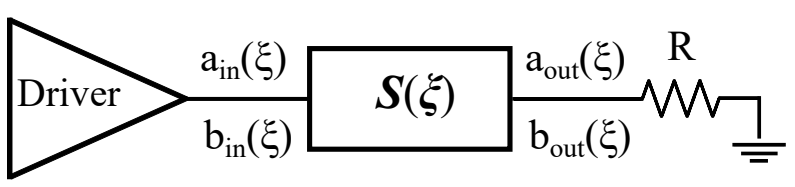

(a)

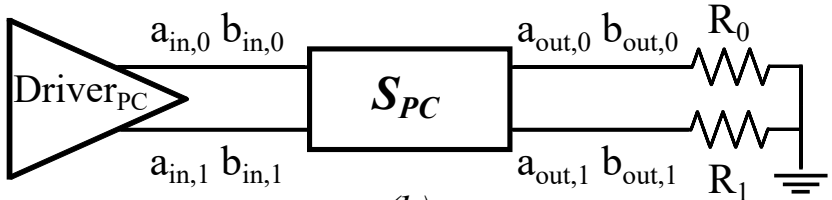

(b)

Fig. 1. (a) Circuit subjected to stochastic variations; (b) Corresponding PC-based deterministic circuit.

where $\boldsymbol{S}$ is the scattering parameter matrix of the stochastic linear system under study. The dependency on the frequency variable $s=j \omega$ in (1) is omitted for the sake of notational conciseness. Following the approach described in [7], it is possible to describe the relationship between the PC coefficients of the incident and reflected waves as

$$
\boldsymbol{b}_{P C}=\boldsymbol{S}_{P C} \boldsymbol{a}_{P C}
$$

where vectors $\boldsymbol{a}_{P C}, \boldsymbol{b}_{P C} \in \mathbb{C}^{(M+1) N \times 1}$ collect the (deterministic) PC-expansion coefficients of the corresponding wave variables, with $M+1$ the number of the PC basis functions considered, whereas $\boldsymbol{S}_{P C} \in \mathbb{C}^{(M+1) N \times(M+1) N}$ is a deterministic matrix obtained from the original stochastic scattering parameters by means of GP.

It is important to remark that $\boldsymbol{S}_{P C}$ can still be regarded as a scattering parameter matrix: the passivity conditions for deterministic scattering parameters still apply. Hence, a stable and passive state-space representation of $\boldsymbol{S}_{P C}$, suitable for time-domain analysis, can be obtained by means of the vector fitting (VF) algorithm [10], [11]. Note that such a state-space model can be converted into an equivalent circuit via a suitable synthesis technique [12].

It is possible to prove that the $p^{\text {th }}$ PC coefficients of the incident $a_{k p}$ and reflected $b_{k p}$ wave at the $k^{\text {th }}$ port of the system depend only on the corresponding PC coefficient of the voltage $V_{k p}$ and current $I_{k p}$ at the same port as

$$
a_{k p}=\frac{V_{k p}+Z I_{k p}}{2 \sqrt{|\Re(Z)|}}, \quad b_{k p}=\frac{V_{k p}-Z^{*} I_{k p}}{2 \sqrt{|\Re(Z)|}}
$$

where $Z$ is the reference impedance, * denotes the transpose and complex conjugate operator, and $p=0, \ldots, M$. Hence, once analogous models are computed for the nonlinear devices attached at the terminations of the linear system described by (2), their connection is straightforward, as illustrated in Fig. 1 for the simple case of a single-input/single-output linear system terminated with a resistor and a nonlinear driver, when two PC basis functions are used in the model. Furthermore, it is important to point out that this modeling approach readily applies to any electrical (e.g., circuit or electromagnetic) system supporting a scattering representation (1).

\section{B. Stochastic Macromodeling of Nonlinear Components}

Nonlinear components are described by suitable relationships between the time-domain counterparts of the port voltages and currents in (3), here denoted as

$$
\boldsymbol{i}(\boldsymbol{\xi}, t)=\mathcal{F}(\boldsymbol{v}(\boldsymbol{\xi}, t), t)
$$

where vectors $\boldsymbol{v}(\boldsymbol{\xi}, t)$ and $\boldsymbol{i}(\boldsymbol{\xi}, t)$ collect all port voltages and currents, whereas $\mathcal{F}$ is a vectorized nonlinear and possibly dynamic operator whose $k^{\text {th }}$ row, $i_{k}(\boldsymbol{\xi}, t)=\mathcal{F}_{k}(\boldsymbol{v}(\boldsymbol{\xi}, t), t)$, describes the nonlinear relationship between the $k^{\text {th }}$ port current and (all) port voltages. It should be noted that the port voltages and currents are stochastic as a result of the variability in the linear part of the network. However, for the sake of simplicity, the nonlinear component itself is considered to be deterministic in this paper.

Replacing the port voltages and currents in (4) with their PC expansions, and enforcing the resulting equation to hold for $M+1$ values of $\boldsymbol{\xi}$, pre-determined via the ST algorithm [1], yields a deterministic system of $M+1$ equations in the voltage and current PC coefficients. For example, for the current at the $k^{\text {th }}$ port:

$$
\begin{aligned}
a_{00} i_{k 0}+\ldots+a_{0 M} i_{k M}= & \mathcal{F}_{k}\left(a_{00} \boldsymbol{v}_{0}+\ldots+a_{0 M} \boldsymbol{v}_{M}\right) \\
& \vdots \\
a_{M 0} i_{k 0}+\ldots+a_{M M} i_{k M}= & \mathcal{F}_{k}\left(a_{M 0} \boldsymbol{v}_{0}+\ldots+a_{M M} \boldsymbol{v}_{M}\right)
\end{aligned}
$$

where $a_{m k}$ are mere scalar coefficients obtained by evaluating the PC basis functions at the ST points. The time variable $t$ in the voltage and current PC coefficients in (5) is omitted for notational convenience. From (5), an explicit relation for the coefficients of the port current is obtained by inverting the system in matrix form:

$\left[\begin{array}{c}i_{k 0} \\ \vdots \\ i_{k M}\end{array}\right]=\left[\begin{array}{ccc}a_{00} & \ldots & a_{0 M} \\ \vdots & \ddots & \vdots \\ a_{M 0} & \ldots & a_{M M}\end{array}\right]^{-1}\left[\begin{array}{c}\mathcal{F}_{k}\left(a_{00} \boldsymbol{v}_{0}+\ldots+a_{0 M} \boldsymbol{v}_{M}\right) \\ \vdots \\ \mathcal{F}_{k}\left(a_{M 0} \boldsymbol{v}_{0}+\ldots+a_{M M} \boldsymbol{v}_{M}\right)\end{array}\right]$

The system (6) has a SPICE-compatible circuit interpretation, as described in [6]. It can be inferred from the vector term in the r.h.s. of (6) that such an equivalent circuit requires $M+1$ replicas of the nonlinear component, and its topology is independent of the specific type of nonlinear element, as long as it has a description in the form of (4).

It is important to note that the deterministic models for both the linear and nonlinear parts of the circuit are $M+1$ times larger in terms of ports number with respect to the original stochastic counterpart, and they are typically referred to as "augmented systems". However, when connecting such deterministic augmented systems, a single time-domain simulation of the resulting circuit provides all the PC coefficients of port voltages and currents. Indeed, one of the main advantages of PC is that statistical information like stochastic moments 
and probability distribution functions (PDF)/cumulative distribution functions $(\mathrm{CDF})$ are readily obtained from the corresponding PC expansion coefficients [9]. The combination of the two modeling approaches outlined in Sections II-A and II-B provides a powerful design tool that allows for the simulation of an arbitrary combination of stochastic passive linear systems, described by means of their scattering parameters, and nonlinear components.

\section{NUMERICAL EXAMPLE}

In order to validate the proposed stochastic modeling approach, a comparison with respect to the MC analysis and the PC-based technique in [2], which also computes a stable and passive model for the time-domain analysis, is presented in the following. Differently from the proposed method, the technique in [2] can only be applied to study uniform multiconductor transmission lines, since it is based on the computation of stochastic models of the per-unit-length parameters.

Hence, we will consider the six-ports system described in Fig. 2, composed of three identical pairs of interconnected coupled lossy transmission lines; the corresponding scattering parameters have been evaluated in the frequency range [0 - 2] GHz. It is terminated with a nonlinear driver at one of the input ports, and with diodes at the four output ports. The nominal values of the parameters of the coupled transmission lines are shown in Fig. 2. Due to process variations, the thickness and permittivity of the substrate, as well as the gap between the two coupled lines, are assumed as Gaussian distributed random variables with standard deviations $10 \%$ of their nominal values, respectively. The system is driven at port 1 by an I/O transceiver of a $512 \mathrm{Mb}$ Flash memory chip, which is implemented in SPICE by means of an equivalent and efficient behavioral macromodel [13] and generates a pulse with a rise time of $1 \mathrm{~ns}$. Ports 3 to 6 are connected to diodes and linear lumped elements.

A sparse PC expansion is adopted to build compact and SPICE-compatible models for the system under study and its terminations, according to Section II. The PC coefficients of the port signals are obtained by one time-domain simulation of such models in HSPICE. Figure 3 shows the mean and standard deviation of the voltage transmitted to port 3, calculated by means of $\mathrm{MC}$, the new approach proposed in this paper, and the PC-based technique in [2]. Note that the variability of the system causes variations in the rising and falling edges of the data signal. Figure 4 depicts the stochastic crosstalk voltage at port 4 . These results demonstrate very good agreement among the three methods. From the PC expansions, not only the mean and standard deviation, but also the PDF and CDF can be derived. As shown in Fig. 5, the PDF and CDF of the crosstalk voltage at port 6 for the time instant $t=5.63 \mathrm{~ns}$ obtained with the new technique match well with that from MC analysis. The simulation of $10000 \mathrm{MC}$ samples took $38541 \mathrm{~s}$ on a Dell XPS laptop with an Intel(R) Core(TM) i7 processor running at $2.20 \mathrm{GHz}$ and $16 \mathrm{~GB}$ of RAM, while the new approach and the technique in [2] required $153 \mathrm{~s}$ and

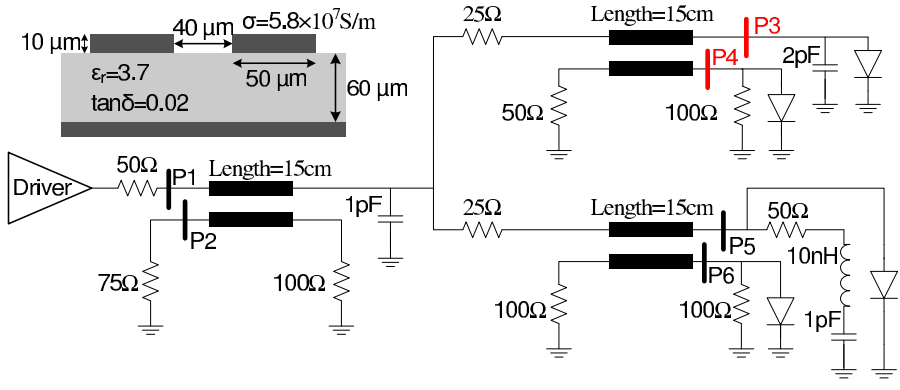

Fig. 2. System under study, composed of three identical pairs of interconnected coupled transmission lines terminated with a nonlinear driver, diodes, and linear lumped elements.
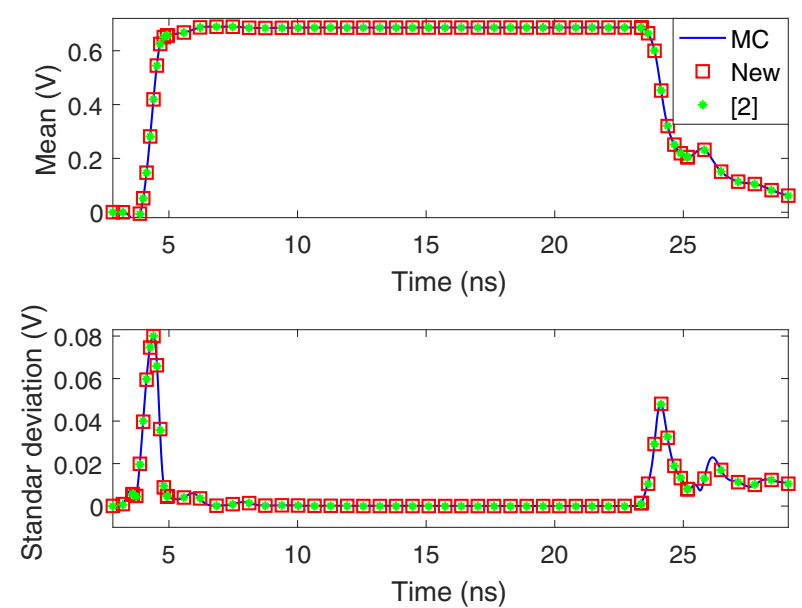

Fig. 3. Mean and standard deviation of the voltage transmitted to port 3. The solid line, and the markers $\square$, * represent the MC, the new technique, and the previous technique in [2], respectively.

$71 \mathrm{~s}$, respectively. The new technique achieves $252 \times$ speedup with regard to MC. For the sake of comparison, the new proposed approach was confronted with the technique in [2], showing similar accuracy. However, it should be noted that the method in [2] is inherently circuit-based, and an equivalent deterministic RLGC-parameter model is implemented for the stochastic transmission lines. Hence, a higher computational efficiency is to be expected when simulating a network that predominantly consists of circuit components only. Yet, in contrast to [2], the new technique is more general and allows including any stochastic passive linear system since it is based on a scattering representation.

\section{CONCLUSION}

This paper focuses on the stochastic macromodeling and simulation of general linear systems with nonlinear terminations. The proposed strategy is based on computing deterministic PC-based augmented systems describing the relationship between the PC coefficients of the port signals of the original system under stochastic variations. These augmented systems can be simulated in any circuital simulator and statistical features are readily obtained by means of only one time-domain 

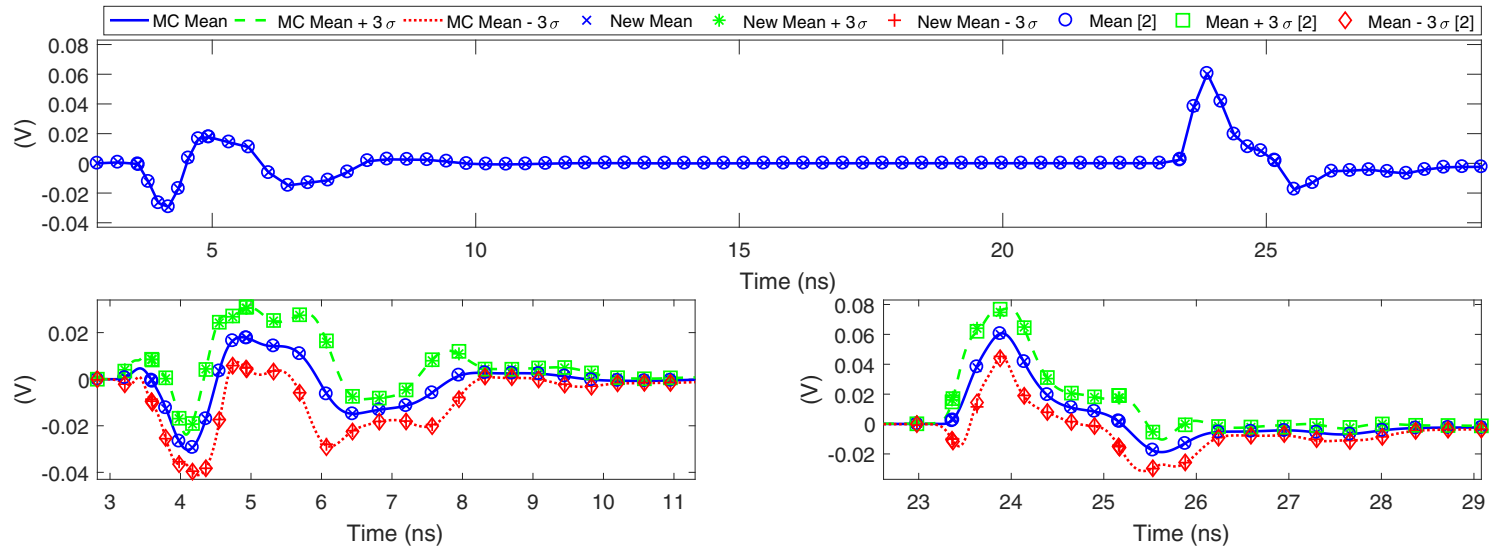

Fig. 4. Top: the mean of the crosstalk voltage at port 4, where the solid line, the symbols $\times$ and $\circ$ represent the MC, the new technique, and the PC-based technique [2], respectively. Bottom: the mean and the $3 \sigma$ range of the same signal at the rise and fall times of the pulse. The (solid, dash, dot) lines are the results from the MC analysis; the markers $(\times, *,+)$ and $(\circ, \square, \diamond)$ represent the new technique and the PC-based technique [2], respectively.

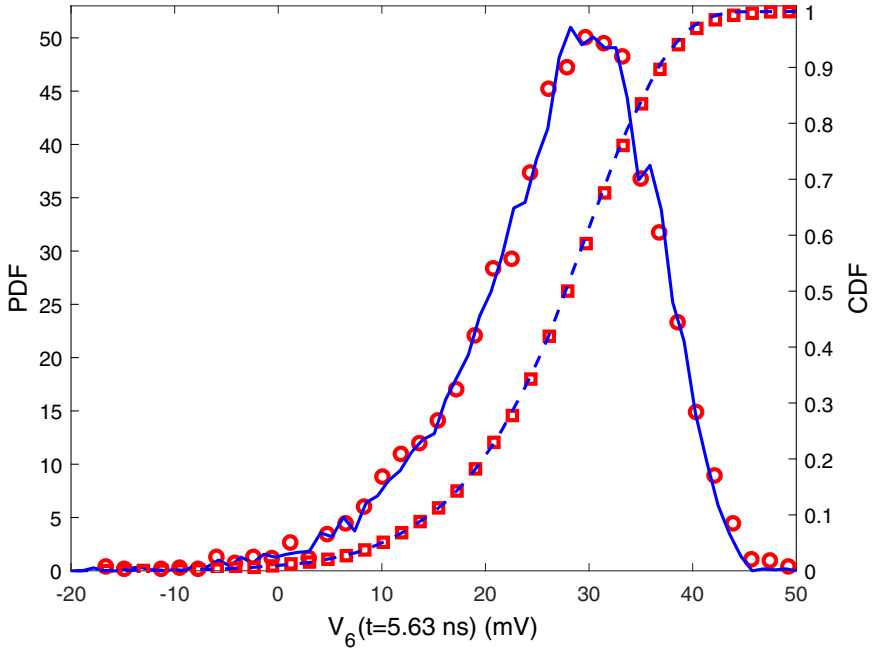

Fig. 5. PDF (full line) and CDF (dashed line) of the crosstalk voltage at port 6 for $t=5.63 \mathrm{~ns}$ computed using the new proposed technique; the circles (o) and squares $(\square)$ represent the same quantities computed by the MC method.

simulation. The proposed approach applies to any stochastic passive linear system with arbitrary nonlinear terminations, thereby extending the scope of applicability of previous PCbased techniques significantly. The accuracy and efficiency of the proposed method are validated by a suitable numerical example, achieving a simulation speedup of $252 \times$ with respect to the MC analysis.

\section{ACKNOWLEDGMENT}

The authors wish to express their gratitude to Prof. Igor Stievano, Politecnico di Torino, for providing the behavioral driver model used in the application example. This work was supported in part by the Research Foundation Flanders (FWOVlaanderen), of which Dr. Manfredi is a Postdoctoral Research Fellow.

\section{REFERENCES}

[1] Z. Zhang, T. A. El-Moselhy, I. M. Elfadel and L. Daniel, "Stochastic testing method for transistor-level uncertainty quantification based on generalized polynomial chaos," IEEE Trans. Comput.-Aided Design Integr. Circuits Syst, vol. 32, no. 10, pp. 1533-1545, Oct. 2013.

[2] P. Manfredi, D. Vande Ginste, D. De Zutter, and F. G. Canavero, "Uncertainty assessment of lossy and dispersive lines in spice-type environments," IEEE Trans. Compon., Packag., Manuf. Technol., vol. 3, no. 7 , pp. 1252-1258, Jul. 2013.

[3] D. Spina, F. Ferranti, T. Dhaene, L. Knockaert, and G. Antonini, "Polynomial chaos-based macromodeling of multiport systems using an inputoutput approach," Int. J. Numer. Model., vol. 28, no. 5, pp. 562581, Sep./Oct. 2015.

[4] M. R. Rufuie, E. Gad, M. Nakhla and R. Achar, "Generalized hermite polynomial chaos for variability analysis of macromodels embedded in nonlinear circuits," IEEE Trans. Compon., Packag., Manuf. Technol., vol. 4, no. 4, pp. 673-684, Apr. 2014

[5] D. Spina, F. Ferranti, G. Antonini, T. Dhaene and L. Knockaert, "Efficient variability analysis of electromagnetic systems via polynomial chaos and model order reduction," IEEE Trans. Compon., Packag., Manuf. Technol., vol. 4, no. 6, pp. 1038-1051, Jun. 2014.

[6] P. Manfredi and F. G. Canavero, "Efficient statistical simulation of microwave devices via stochastic testing-based circuit equivalents of nonlinear components," IEEE Trans. Microw. Theory Techn., vol. 63, no. 5, pp. 1502-1511, May 2015.

[7] D. Spina, T. Dhaene, L. Knockaert and G. Antonini, "Polynomial chaosbased macromodeling of general linear multiport systems for timedomain analysis," in print on IEEE Trans. Microw. Theory Techn., 2017.

[8] D. Xiu and G.M. Karniadakis, "The Wiener-Askey polynomial chaos for stochastic differential equations," SIAM J. Sci. Comput., vol. 24, no. 2, pp. 619-644, Apr. 2002.

[9] M. S. Eldred, "Recent advance in non-intrusive polynomial-chaos and stochastic collocation methods for uncertainty analysis and design," in Proc. 50th AIAA/ASME/ASCE/AHS/ASC Struct., Structural Dynam., Mat. Conf., AIAA-2009-2274, Palm Springs, California, May 2009.

[10] B. Gustavsen and A. Semlyen, "Rational approximation of frequency domain responses by vector fitting," IEEE Trans. Power Del., vol. 14, no. 3, pp. 1052-1061, Jul. 1999.

[11] D. Deschrijver, and T. Dhaene, "Fast passivity enforcement of Sparameter macromodels by pole perturbation," IEEE Trans. Microw. Theory Techn., vol. 57, no. 3, pp. 620-626, Mar. 2009.

[12] R. Neumayer, F. Haslinger, A. Stelzer and R. Weigel, "Synthesis of SPICE-compatible broadband electrical models from n-port scattering parameter data," in Proc. IEEE Int. Symp. on Electromagnetic Compatibility (EMC), Minneapolis, USA, 2002.

[13] I. S. Stievano, L. Rigazio, F. G. Canavero, T. R. Cunha, J. C. Pedro, H. M. Teixeira, A. Girardi, R. Izzi, and F. Vitale, "Behavioral modeling of ic memories from measured data," IEEE Trans. Instrum. Meas., vol. 60, no. 10, pp. 3471-3479, Oct. 2011. 